\title{
Highly efficient optical filter based on vertically coupled photonic crystal cavity and bus waveguide
}

\author{
Kapil Debnath, ${ }^{1, *}$ Karl Welna, ${ }^{1}$ Marcello Ferrera, ${ }^{1}$ Kieran Deasy, ${ }^{2}$ David G. Lidzey, ${ }^{2}$ and Liam O’Faolain ${ }^{1}$ \\ ${ }^{1}$ School of Physics and Astronomy, University of St Andrews, North Haugh, St Andrews KY16 9SS, UK \\ ${ }^{2}$ Department of Physics and Astronomy, University of Sheffield, Sheffield S3 7RH, UK \\ *Corresponding author: kd343@st-andrews.ac.uk
}

Received October 5, 2012; revised December 3, 2012; accepted December 8, 2012; posted December 11, 2012 (Doc. ID 177492); published January 9, 2013

\begin{abstract}
We experimentally demonstrate a new optical filter design based on a vertically coupled photonic crystal (PhC) cavity and a bus waveguide monolithically integrated on the silicon-on-insulator platform. The use of a vertically coupled waveguide gives flexibility in the choice of the waveguide material and dimensions, dramatically lowering the insertion loss while achieving very high coupling efficiencies to wavelength scale resonators and thus allows the creation of PhC-based optical filters with very high extinction ratio ( $>10 \mathrm{~dB})$. (c) 2013 Optical Society of America OCIS codes: $230.3120,230.3990,230.5750,230.5298$.
\end{abstract}

Wavelength division multiplexing (WDM) has become an essential part of modern optical communication systems, maximizing the available bandwidth by simultaneously transmitting multiple channels through the same optical waveguide using multiple wavelengths. For different WDM functions, such as optical filtering [1] $]$, modulation [2, $\underline{3}]$, multiplexing/demultiplexing [3] , and routing [4] , optical waveguide-resonator systems have been studied extensively as, due to their small footprint, they require very low operating power and promise chip-scale integration.

Optical resonators can be classified into two categories [5], namely traveling-wave, such as the microring resonator and microdisk resonator, and standing-wave resonators, such as photonic crystal (PhC) cavities. Optical filters based on traveling-wave resonators, where both the resonator and waveguide are monolithically fabricated on the same platform, have been the subject of a large volume of recent research. The primary reasons behind their popularity are their high extinction ratio and high quality factor. However, their free spectral range restricts the scalability of such devices in terms of the number of channels.

On the other hand, the design of PhC cavities with small mode number (i.e., single-mode operation) and ultrahigh quality factor is relatively mature [므, $]$ ]; nevertheless, due to their small size, efficient coupling of light in and out of the cavity is challenging, making the overall extinction ratio of such devices low. The conventional approach is to use a PhC line-defect waveguide side-coupled to a $\mathrm{PhC}$ cavity, but the line defect itself introduces additional Fourier components into the light cone of the cavity, which in turn reduces the overall extinction ratio [8].

In [1], a different optical filter design was proposed where a silicon bus waveguide is vertically coupled to a silicon PhC cavity. Here we develop insights into such optical filters and show that very efficient transfer of light can be achieved even between modes with dissimilar modal refractive indices, thus achieving an optical filter with a high extinction ratio. As a consequence, lowrefractive-index waveguides with large mode areas may be used, allowing very efficient interfacing with optical fibers. In addition, the monolithic vertically coupled geometry allows easy cascading of multiple cavities, making it very promising for WDM applications.
Our vertical coupling scheme is shown in Fig. 1 . A bus waveguide is placed vertically above a silicon $\overline{\mathrm{PhC}}$ cavity. An oxide barrier layer between the cavity and the waveguide works as a physical separation between the two optical modes. At the resonance wavelength of the cavity, light couples from the waveguide mode to the cavity mode. Under the weak coupling approximation, the transmission and reflection in the waveguide at resonance can be expressed as [5]

$$
T=\frac{Q_{\text {total }}^{2}}{Q_{\text {cavity }}^{2}}, \quad R=\frac{Q_{\text {total }}^{2}}{Q_{\text {coupling }}^{2}} .
$$

The cavity $Q$ factor, $Q_{\text {cavity }}$, is given by $1 / Q_{\text {cavity }}=$ $1 / Q_{\text {design }}+1 / Q_{\text {fabrication, where }} Q_{\text {design }}$ is given by the cavity design and $Q_{\text {fabrication }}$ by fabrication imperfections. $Q_{\text {coupling }}$ depends on the coupling efficiency between the bus waveguide and cavity mode. The overall $Q$ factor of the system $Q_{\text {total }}$ is

$$
\frac{1}{Q_{\text {total }}}=\frac{1}{Q_{\text {cavity }}}+\frac{1}{Q_{\text {coupling }}} .
$$

It is clear from the above equations that in order to achieve a large drop in transmission, that is $T \sim 0$, and large reflection, that is $R \sim 1$ at resonance $Q_{\text {design }}$, $Q_{\text {fabrication }} \gg Q_{\text {total }}$. In other words, we need to increase the coupling efficiency while maintaining a high quality factor for the cavity $\left(Q_{\text {cavity }}\right)$.

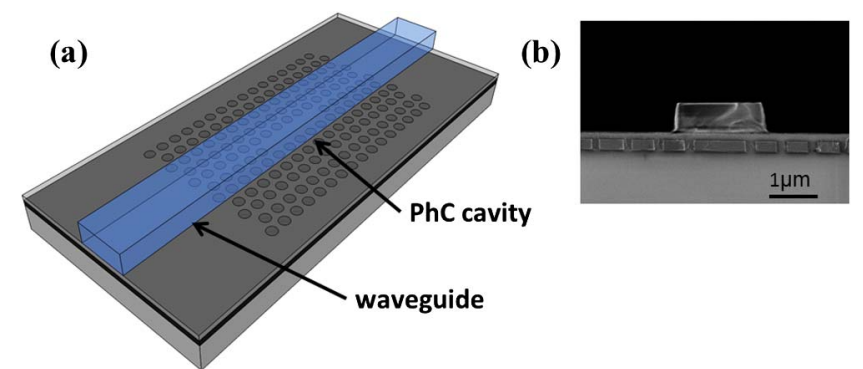

Fig. 1. (Color online) (a) Schematic diagram of the optical filter where a bus waveguide is vertically coupled to a PhC cavity. (b) Scanning electron microscopy image of the device cross-section at the cavity region. 
In-plane bus waveguide-cavity coupling configurations, which simplistically might be expected to affect only $Q_{\text {coupling, }}$, actually have an impact on $Q_{\text {cavity }}$ by disturbing the cavity structure and introducing additional $K$-space field components inside the light cone. Threedimensional finite-difference time-domain simulations have shown that placing an in-plane waveguide three rows away from an unmodified L3 cavity reduces $Q_{\text {cavity }}$ from 4982 to 2690 . Hence, simultaneously achieving high coupling efficiency and maintaining high $Q_{\text {cavity }}$ becomes difficult. In contrast, when the waveguide is placed vertically, the presence of the waveguide does not significantly disturb the lattice structure of the PhC cavity, and as a result a high $Q_{\text {cavity }}$ can be maintained.

The coupling efficiency depends mainly on two factors: (1) the spatial overlap of the evanescent modes of the cavity and the waveguide and (2) $k$-vector matching between the two modes. The spatial overlap between the two modes can be optimized simply by controlling the thickness of the barrier layer. To ensure $k$-vector matching between the two optical modes, similar materials are used to guide both the optical modes in conventional coupled systems, such as directional couplers. In the case of $\mathrm{PhC}$ cavities, the $K$-space distribution is expanded due to their ultrasmall mode volume, helping achieve matching with the optical mode of a waveguide with dissimilar material.

Figure 2 shows the real-space and $K$-space distribution of the waveguide mode and the first-order mode of the cavity used in this Letter (the design was introduced in [7]). Due to the rich $K$-space distribution of the cavity mode, a small change in the effective index of the waveguide can cause a dramatic change in the mode overlap and hence a large change in the transmittance of the whole system. Therefore, the coupling efficiency between the waveguide mode and the cavity mode can be controlled by changing either the physical properties of the waveguide, such as the dimensions or material, the lattice structure of the $\mathrm{PhC}$ cavity, or the thickness of the barrier layer.

We fabricated the PhC cavity in a silicon-on-insulator platform [9]. The cavity was created by locally modifying the width of a W1 $\mathrm{PhC}$ waveguide. The $\mathrm{PhC}$ was a triangular lattice of oxide holes with radius $r=95 \mathrm{~nm}$ and lattice constant $a=390 \mathrm{~nm}$. The $\mathrm{PhC}$ area was (a)

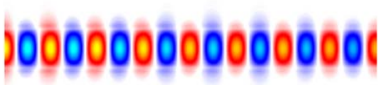

(c)

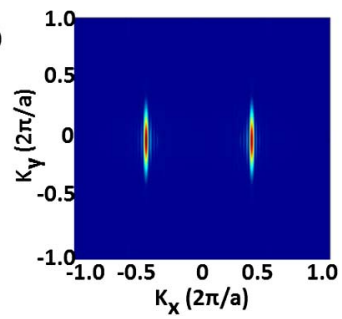

(b)

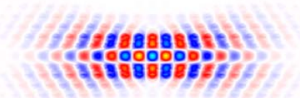

(d)

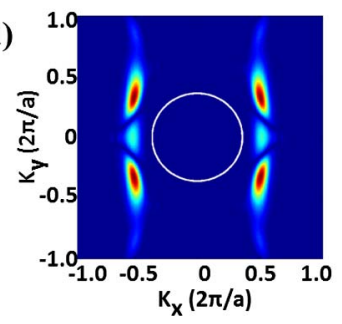

Fig. 2. (Color online) Dominant electric field profile of (a) waveguide; (b) cavity; $K$-space distribution of the dominant field; (c) waveguide; and (d) cavity.
$10 \mu \mathrm{m} \times 20 \mu \mathrm{m}$. The sample was then coated with a flowable oxide (Dow Corning FOx), which fills the PhC holes and acts as the buffer layer. After curing, the FOx has a refractive index similar to that of buried oxide $(\sim 1.4)$. A thickness of $200 \mathrm{~nm}( \pm 10 \mathrm{~nm})$ was used to achieve efficient coupling while keeping $Q_{\text {total }}$ reasonably high. To demonstrate the full versatility of our coupling technique, we have used two different materials for the top waveguide: ZEON ZEP $520 \mathrm{~A}(n \approx 1.52)$ and $\mathrm{Si}_{3} \mathrm{~N}_{4}$ $(n \approx 1.88)$. The waveguides were patterned in the ZEP layer using e-beam lithography, giving a cross section of $3 \mu \mathrm{m} \times 2 \mu \mathrm{m}$. For the $\mathrm{Si}_{3} \mathrm{~N}_{4}$ waveguides, a $500 \mathrm{~nm}$ layer of $\mathrm{Si}_{3} \mathrm{~N}_{4}$ was deposited using plasma-enhanced chemical vapor deposition, and waveguides were then created in $\mathrm{Si}_{3} \mathrm{~N}_{4}$ layer using e-beam lithography and dry etching.

Figure 3 shows the transmission and reflection spectra (measure $\bar{d}$ using an end-fire setup) of an optical filter based on coupled cavity-waveguide system at the resonance wavelength of the first-order mode of the $\mathrm{PhC}$ cavity, where the waveguide material was ZEP 520A. A quasi-TE mode was used for all the measurements. At resonance, the extinction ratio is almost $7 \mathrm{~dB}$ while the overall quality factor is maintained at 50,000. From these results and using Eqs. (1) and (2), we can infer that $Q_{\text {coupling }} \approx 60,000$ and $Q_{\text {cavity }} \approx 110,000$.

The ZEP-based waveguides were preliminarily used to demonstrate the low fiber-to-fiber loss $(<3 \mathrm{~dB})$ of our optical filter. Subsequently, $\mathrm{Si}_{3} \mathrm{~N}_{4}$ waveguides of varied width ( $\sim 6 \mathrm{~dB}$ insertion loss) were used to demonstrate the effect of the $K$-space overlap on the coupling efficiency and thereby the overall extinction ratio of the filter and $Q_{\text {cavity }}$. This change in physical dimension varies the effective index and thereby moves the Fourier components of the waveguide mode along $K_{x}$ in $K$-space; as a result the overlap intensity with the cavity mode also changes. The widths of the $\mathrm{Si}_{3} \mathrm{~N}_{4}$ waveguides we used range from 0.8 to $1.5 \mu \mathrm{m}$. In most of this range the waveguides support a single quasi-TE mode. However, the higher-order mode that occurs for widths $>1.2 \mu \mathrm{m}$ accounts for very little energy compared with the fundamental mode and does not have a $K$-space overlap with the cavity modes. For these reasons, only the fundamental waveguide mode is taken into account in our analysis.

The PhC cavity design used here is similar to that used in the previous experiment (Fig. 1). A design was chosen that exhibits two high- $Q$ modes so as to highlight the

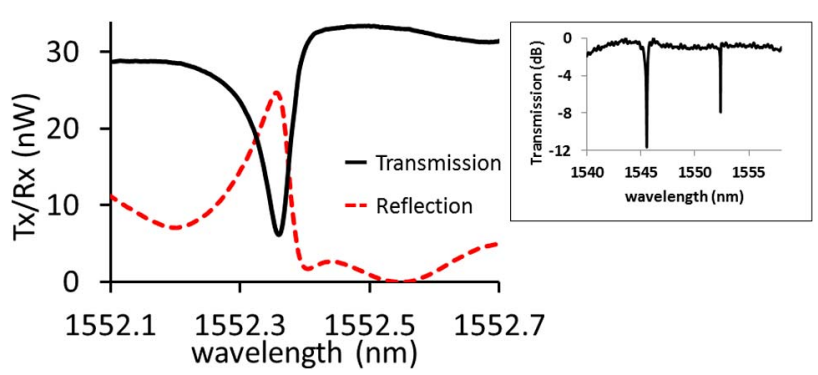

Fig. 3. (Color online) Transmission and reflection spectra of a vertically coupled cavity waveguide (ZEP) system. The extinction ratio is measured as the ratio between the on- and offresonance transmission. The inset shows the first two modes of the cavity. 


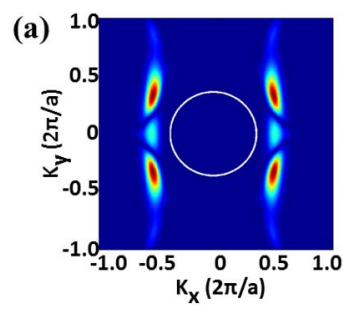

(c)
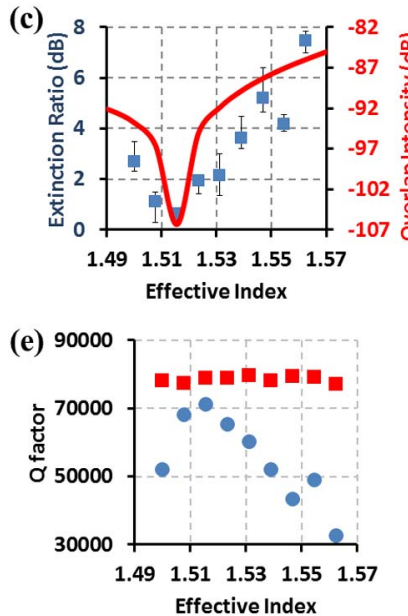
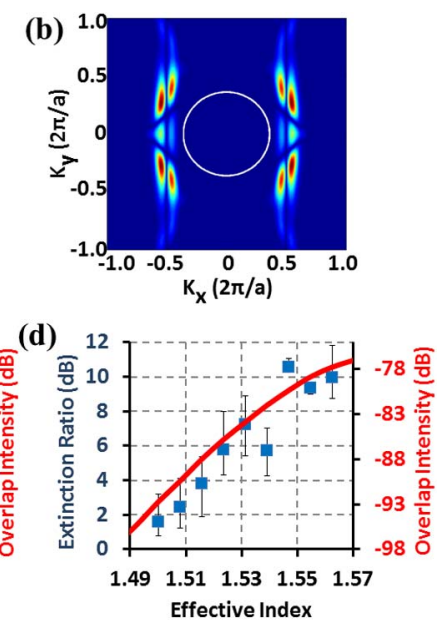

(f)

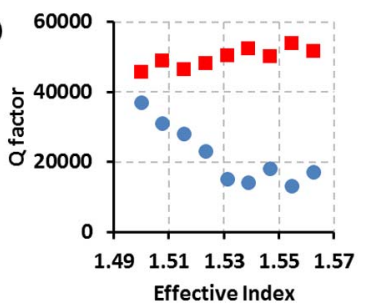

Fig. 4. (Color online) $K$-space distribution of dominant electric field of (a) mode1 and (b) mode2. Extinction ratio (blue squares) and $K$-space overlap intensity (red curve) for different waveguide dimensions for (c) mode1 and (d) mode2. $Q_{\text {total }}$ (blue circles) and $Q_{\text {cavity }}$ (red squares) for different waveguide dimensions for (e) mode1 and (f) mode2.

importance of the $K$-space overlap. Figures $\underline{4(a)}$ and $4(\mathrm{~b})$ show the $K$-space distribution of the first two modes of the cavity. In Figs. 4(c) and 4(d), the solid curves are the calculated $K$-space overlap intensities as the effective index of the waveguide is modified, and the blue squares show the corresponding measured extinction ratios. Comparing the theoretical overlap intensities with experimental extinction ratios, we note that both shapes match well. In Figs. 4(e) and 4(f), blue circles represents $Q_{\text {total }}$ and red squares represents $Q_{\text {cavity }}$, which is calculated using Eq. (1). As one can see, due to different coupling efficiencies the $Q_{\text {total }}$ is different for different

waveguides, but $Q_{\text {cavity }}$ remains almost invariant to any change in coupling efficiency, thus providing a very high extinction ratio; see Eq. (1). Due to fabrication variations, $Q_{\text {cavity }}$ is slightly smaller than that measured in the previous experiment (Fig. 1).

In this work an optical filter comprising a vertically coupled cavity-waveguide system is proposed and experimentally demonstrated, providing an efficient, ultracompact filter. This vertical coupling technique allows complete flexibility in choosing the cavity and waveguide design and achieves high $Q$ factors and coupling efficiencies. With increases in $Q_{\text {cavity }}\left(10^{6}\right.$ possible [10] $), 20 \mathrm{~dB}$ extinction ratios for $Q_{\text {total }} \sim 10^{5}$ are possible. Furthermore, the use of low-index waveguides ensures very low insertion loss for such devices. This is a very promising platform for the realization of key functions, such as optical modulation [9] and filtering.

This work is supported by EPSRC under the UK silicon photonics project and through the EraNET Nano-Sci project LECSIN. Kieran Deasy and David Lidzey also acknowledge financial support from the Marie Curie ITN: ICARUS. The authors acknowledge Andrea di Falco for useful discussions.

\section{References}

1. M. Qiu, Opt. Lett. 30, 1476 (2005).

2. T. Tanabe, K. Nishiguchi, E. Kuramochi, and M. Notomi, Opt. Express 17, 22505 (2009).

3. Q. Xu, B. Schmidt, J. Shakya, and M. Lipson, Opt. Express 14, 9430 (2006).

4. N. Sherwood-Droz, H. Wang, L. Chen, B. G. Lee, A. Biberman, K. Bergman, and M. Lipson, Opt. Express 16, 15915 (2008).

5. Y. Xu, Y. Li, R. K. Lee, and A. Yariv, Phys. Rev. E 62, 7389 (2000).

6. B.-S. Song, S. Noda, T. Asano, and Y. Akahane, Nat. Mater. 4, 207 (2005).

7. K. Welna, S. L. Portalupi, M. Galli, L. O'Faolain, and T. F. Krauss, IEEE J. Quantum Electron. 48, 1177 (2012).

8. Z. Zhang and M. Qiu, Opt. Express 13, 2596 (2005).

9. K. Debnath, L. O'Faolain, F. Y. Gardes, A. G. Steffan, G. T. Reed, and T. F. Krauss, Opt. Express 20, 27420 (2012).

10. S.-W. Jeon, J.-K. Han, B.-S. Song, and S. Noda, Opt. Express 18, 19361 (2010). 